\title{
Impact of COVID-19: urging a need for multi-domain assessment of COVID-19 inpatients
}

\author{
Arne Heyns $^{1} \oplus$. Jolan Dupont ${ }^{2} \cdot$ Evelien Gielen $^{2,3} \cdot$ Johan Flamaing $^{2,3} \cdot$ Koen Peers $^{1,4} \cdot$ Rik Gosselink $^{5} \cdot$ Bart Vrijsen $^{1,5}$. \\ Natalie Lorent ${ }^{6}$. Stephanie Everaerts ${ }^{6} \cdot$ Wim Janssens $^{3,6}$. Greet Hermans ${ }^{7,8} \cdot$ Kim Caluwé $^{1,5} \cdot$ Blanche Staes $^{1}$. \\ Ann Goeleven ${ }^{9} \cdot$ Ann Meulemans $^{10} \cdot$ Hadi Waelkens ${ }^{11} \cdot$ Hilde Beyens $^{1} \cdot$ Jos Tournoy $^{2,3}$
}

Received: 28 January 2021 / Accepted: 15 March 2021 / Published online: 30 March 2021

(c) European Geriatric Medicine Society 2021

\section{Key summary points}

Aim To retrospectively analyse data obtained from the multi-domain assessment of hospitalized COVID-19 patients, to describe their health status at discharge, and to investigate whether subgroups of patients, more specific ICU patients and older adults ( $>70$ years), had more (or less) risk to experience specific impairments.

Findings The results of the assessment show that physical, functional, cognitive, nutritional, and psychological impairments are highly prevalent in the group of COVID-19 patients, both in ICU and non-ICU patients, adults and older adults.

Message The high prevalence of physical, cognitive, psychological, and functional impairments in hospitalized COVID-19 patients, both ICU and non-ICU patients, indicates that assessment of impairments is imperative.

\begin{abstract}
Objective To retrospectively analyse data obtained from the multi-domain assessment of hospitalized COVID-19 patients, to describe their health status at discharge, and to investigate whether subgroups of patients, more specific ICU patients and older adults ( $>70$ years), had more (or less) risk to experience specific impairments.

Methods Retrospective case series in the University Hospitals Leuven, Belgium of confirmed COVID-19 patients 'after surviving an ICU-stay', 'aged $\geq 70$ years', or 'aged $<70$ years with a length of hospitalization $>7$ days'. Exclusion criteria were 'unwilling to cooperate', 'medically unstable', or 'palliative care policy'. Following tests were used: 'Five Times Sit To Stand Test', 'hand grip dynamometry', 'Barthel index', 'Swallowing screening', 'Montreal Cognitive Assessment', 'Hospital Anxiety and Depression Scale', and 'Nutritional Risk Screening 2002'.

Results One or more tests were obtained in $135 / 163$ patients $(83.3 \%)$. Physical impairments were present in $43.2-82.8 \%$ of the patients. Median BI was 10/20 indicating limited self-dependency. Swallow impairments were present in 3/53 (5.7\%) and 24/76 (31.6\%) had risk of malnutrition. Impaired memory was seen in 26/43 (60.5\%) and 22/47 (46.8\%) had elevated
\end{abstract}

Arne Heyns

arneheyns@hotmail.com

1 Department of Physical and Rehabilitation Medicine, University Hospitals Leuven, Leuven, Belgium

2 Department of Geriatric Medicine, University Hospitals Leuven, Leuven, Belgium

3 Department of Public Health and Primary Care, KU Leuven, Leuven, Belgium

4 Department of Development and Regeneration, KU Leuven, Leuven, Belgium

5 Department of Rehabilitation Sciences, KU Leuven, Herestraat 49, 3000 Leuven, Belgium

6 Respiratory Division, University Hospitals Leuven, Leuven, Belgium
7 Department of Cellular and Molecular Medicine, KU Leuven, Herestraat 49, 3000 Leuven, Belgium

8 Medical Intensive Care Unit, Department of General Internal Medicine, University Hospitals Leuven, Herestraat 49, 3000 Leuven, Belgium

9 Research Group of Experimental Oto-Rhino-Laryngology, KU Leuven, Leuven, Belgium

10 Department of Chronic Diseases, Metabolism and Ageing, KU Leuven, Leuven, Belgium

11 KU Leuven, University Hospitals Leuven, University Psychiatric Center, Adult Psychiatry, 3000 Leuven, Belgium 
anxiety/depression scores. Older adults had more physical, functional, and cognitive impairments. ICU patients had a lower hand grip force.

Conclusion(s) The high prevalence of physical, cognitive, psychological, and functional impairments in hospitalized COVID19 patients, both ICU and non-ICU patients, indicates that assessment of impairments is imperative. These results imply that rehabilitation and follow-up is essential for these patients. This paper proposes a short, workable assessment composed with known outcome measures to assess different domains of COVID-19 patients.

Keywords Assessment · COVID-19 · Post-infection · Cognitive impairment · Physical impairment · Nutritional impairment $\cdot$ Rehabilitation $\cdot$ Impairment

\section{Introduction}

Coronavirus 19 disease (COVID-19) created an unforeseen pandemic afflicting a large variety of patients with and without known comorbidities, and led to a high rate of hospitalizations [1]. The peak in Belgium occurred in the beginning of April 2020 (5590 patients hospitalized and 1285 of them admitted to on an intensive care unit (ICU) on the 8th of April). Between 15th of March 2020 and 11th of June $2020,17.568$ COVID-19 patients $(=0.15 \%$ of the Belgian population [2]) were hospitalized in Belgium [3]. This high hospitalization rate, both on ICU and non-ICU, had a great impact on regular hospital care. Furthermore, both epidemiological (rapid spread, regional differences, etc.) and logistic (separate pathways for COVID-19 patients, balance between reducing contacts but providing adequate care, tension between social reinsertion and quarantine, etc.) factors created an additional challenge for health care systems, including rehabilitation services [4].

The combination of a high hospitalization rate and limitations caused by isolation and logistic factors made the assessment of different health domains of patients in the acute phase more complex. However, an assessment of the functional status is essential for multiple reasons. First, to evaluate which impairments would arise due to this new unknown disease. Indeed, as an unknown disease with unknown consequences, it was difficult to predict which impairments would arise among the infected patients requiring screening of multiple domains. Second, to determine whether or not a patient could be safely discharged. Third, even more important, to identify patients who would benefit from additional care for recovery of the diagnosed impairments. Finally, to evaluate, when additional care was required, which type of care was recommended (e.g. inpatient rehabilitation, outpatient rehabilitation, pulmonary rehabilitation, psychiatric or psychological follow-up, cognitive training, etc.). Specific attention for ICU patients and older adult patients ( $\geq 70$ years) was recommended as both ICU patients and older adults were expected to have a substantial need for rehabilitation [5, 6]. COVID-19 patients hospitalized at the ICU are at risk of developing ICU-acquired muscle weakness (ICU-AW) [7] while older adult patients are more at risk of developing severe symptoms when infected with COVID-19 [6]. When developing this assessment, it was crucial to accept limitations caused by isolation and logistic problems to increase feasibility.

The need for a pre-discharge assessment was prominent, as illustrated by the survey launched by the International Society of Physical and Rehabilitation Medicine to develop a Clinical Functioning Information Tool [8] specifically for COVID-19 [9] and the European Respiratory Society and American Thoracic Society Task force on Interim Guidance on Rehabilitation in the Hospital and Post-Hospital Phase [10].

At the beginning of the pandemic no pre-existing assessment schedule was available. We developed a short and easy to use, multi-domain assessment in our hospital to evaluate the functional status of the hospitalized COVID-19 patients with a special focus, but not limited to, ICU patients and older adults. The primary goal of this assessment was to screen possible impairments in physical, functional, psychological, nutritional, or cognitive domains. The secondary goal was to identify additional rehabilitation needs based on that information.

The primary objective was to retrospectively analyse the data obtained from the multi-domain assessment of hospitalized COVID-19 patients in the University Hospitals Leuven, Belgium and to describe their health status at hospital discharge. The secondary objective was to investigate whether subgroups of patients, more specific ICU patients and older adults ( $>70$ years), had more (or less) risk to experience specific impairments. This manuscript also provides information about this multi-domain assessment.

\section{Methods}

\section{Patient selection}

Patients with confirmed COVID-19 infection admitted to the University Hospitals Leuven, Belgium, between the 24th of April, 2020 and the 11th of June, 2020 were eligible for the assessment. Diagnosis of COVID-19 was based on a positive nasopharyngeal or lower respiratory tract sample 
polymerase chain reaction test or a combination of upper or lower respiratory infection symptoms (cough, fever, dyspnoea, desaturation) with chest CT findings compatible with COVID-19 [11]. The assessment was performed in all patients diagnosed with COVID-19 who were either admitted to the ward after surviving an ICU-stay, aged $\geq 70$ years (described in this paper as older adults), or aged $<70$ years with a length of hospitalization $>7$ days. Exclusion criteria were unwillingness to cooperate, medical instability, or a palliative care policy. Patients were assessed when considered eligible for discharge from the regular (non-ICU) COVID ward unless specified otherwise.

Approval of the Ethical Committee Research UZ/KU Leuven was obtained for this retrospective analysis (S-number 64591). The need for an informed consent of participants was waived by the Ethical Committee.

\section{Development and content of the assessment battery}

The assessment battery needed to assess different aspects of the patient's function. A multidisciplinary panel of experts, comprising of physiatrists, geriatricians, pulmonologists, psychiatrists, physiotherapists, occupational therapists, dieticians, psychologists, nurses, speech language pathologists, operational directors, and project coordinators was created in the beginning of April 2020. The assessment battery was based on expert consensus.

The expert panel discussed which outcome measures and test to use. The latter required a minimum of materials, minimal additional workload and patient-caregivers contacts, and was to be performed in the patients' room. The final version of the assessment was completed on the 20th of April, 2020 and testing started on the 24th of April, 2020.

\section{Assessments}

The tests were performed by different members of the regular health care providers of the patients. All of them having pre-existing expertise in performing these tests. The following tests were used for the specific domains:

- Physical domain: Five Times Sit To Stand Test (FTSTST) [12] and the hand grip dynamometry [13] were assessed by the physiotherapists;

- Functional domain: Barthel index (BI) [14] as assessed by the occupational therapists and nurses; Swallowing screening according to our own hospital specific protocol, conform international guidelines [15-17] assessed by nurses;

- Cognitive domain: Montreal Cognitive Assessment (MoCA) [18] was performed by the occupational therapists;
- Psychological domain: Hospital Anxiety and Depression Scale (HADS) [19] was taken by the occupational therapists;

- Nutritional domain: Nutritional Risk Screening 2002 (NRS 2002) [20] performed by the nursing staff.

\section{Physical domain}

The hand grip strength was assessed using a Jamar Hand Dynamometer (Patterson Medical Ltd, Nottinghamshire, Great Britain) according to a standardized protocol which was already implemented at the ICU [13]. To facilitate clinical interpretation, the measured values of the hand grip force (HGF) were compared to normative values (percentile 50 value) according to age and gender [21] to obtain a percentage. A percentage of $<80 \%$ of the predictive value was considered abnormal. This cut-off was based on expert opinion with the knowledge that $80 \%$ is often used as a cut-off for abnormality, especially in pulmonary testing [22].

For the FTSTST, patients were instructed to stand up and sit back down five times after each other as quickly as possible with their arms folded across their chest. The time was recorded from the start to the time the patient was seated again for the fifth time. If the patient was not able to perform the test (e.g. need to use the arms, etc.), this was noted as 'unable to perform the test correctly' without registration of the time. A time of $>14 \mathrm{~s}$ or the inability of performing the test correctly was considered abnormal [12, 23].

\section{Functional domain}

The BI was assessed by the nurses (feeding, bathing, grooming, dressing, bowel control, bladder control, and toilet use) and the occupational therapist (transfers, mobility, and stairs). The BI ranged from 0 (total dependence) to 20 (total independence). There was no cut-off score for the BI.

The swallowing screening was performed within $48 \mathrm{~h}$ of admission to the ward. In contrast to the other tests that were executed once patients were eligible for discharge from the COVID normal care unit. Additional exclusion criteria specifically for this test were 'nil per os policy' or 'presence of a gastrostomy tube'. The test consisted of two steps. The first step was assessment of eight non-swallow parameters, indicative for potential or premorbid swallowing problems. In the second step, an actual water swallow test (teaspoon and cup drinking) was performed while nurses checked for swallowing activity and aspiration signs. Progression to the next step was only possible if the previous step was normal. If something was aberrant during one of the steps, the screening assessment was stopped, considered 'impaired'. 


\section{Cognitive domain}

A specific exclusion criterium for the MOCA was 'known cognitive impairment or dementia' mentioned in the patients' file. This was then noted as 'known cognitive impairment'. The MoCA blind was assessed if a patient suffered from visual impairments. A score of $<26$ on the MoCA or $<18$ on the MoCA blind was considered abnormal[18].

\section{Nutritional domain}

The first part of the NRS 2002 was assessed by the nurses within $48 \mathrm{~h}$ after admission. Like the swallow screening, this was in contrast to other tests which were performed shortly before discharge. This part comprises of four questions. The test was scored 'risk of malnutrition' if one or more of those questions were answered with 'yes' or if the BMI of the patient was below 18.5 for patients aged $<70$-years-old or below 20.5 for patients $\geq 70$-years-old.

\section{Psychological domain}

The HADS was not assessed when patients had known cognitive problems or when the occupational therapists judged that the questionnaire could not be completed in a reliable way. The HADS was considered aberrant when the HADSanxiety (HADS-A) or HADS-depression (HADS-D) subscales had a score $>7$, indicating possible anxiety or depression [19].

\section{Data extraction}

We collected the following data from the electronical patient file: date of birth (age), gender, admission to ICU, score on HGF, FTSTST, BI, swallow screening, NRS 2002, HADS, and MoCA.

Data were extracted from the patients' records to an Excel database which was pseudonymised. Data were stored on the secure server of the University Hospitals Leuven, Leuven, Belgium.

\section{Data analysis}

All patients with one or more results of the assessment test battery available were included. Data were analysed retrospectively. Subgroup analysis was performed between ICU and non-ICU populations as well as between those aged $<70$ years and those $\geq 70$ years.

Continuous variables were summarized with standard descriptive statistics, normally distributed data were noted as average \pm SD while non-normally distributed dated were noted as median with interquartile $25 \%$ and $75 \%$ ranges [Q1-Q3]. Categorical variables were summarized with frequencies and percentages. Ninety-five percent confidence intervals (CI) were provided for descriptive statistics.

Shapiro-Wilk test was used to check for normality, Levene's test was used to assess equality of variance. Fisher exact test was performed to analyse different subgroups, independent $t$ test was used to compare means, and Mann-Whitney $U$ test to assess distribution between groups. Odds ratio (OR) was calculated using logistic regression. Data analysis was performed using SPSS, version 26 (IBM Corp. Released 2019. IBM SPSS Statistics for Windows, Version 26.0. Armonk, NY: IBM Corp).

\section{Results}

Out of the 163 patients admitted to a COVID-19 wards in the University Hospitals Leuven between April 24, 2020 and June 11, 2020, 33 (20.2\%) were initially hospitalized on the ICU. One or more tests of the assessment were obtained in 135 of the 163 patients ( $83.3 \%$ ). There were 68 females (50.4\%) and 67 males (49.6\%). The median age of the 135 patients was 72.0 years [58.0-86.0]. The median age of ICU patients was 60.0 year [IQR 51.5-67.5], which was significantly lower than those admitted on the non-ICU ward, 77.0 year [IQR 63.0-88.0], $p<0.0001$.

Table 1 shows how many patients were assessed for each specific test. Some patients were eligible for assessment, but were not or incompletely assessed due to limited period of time before discharge, limited supply of protective personal equipment or other logistic problems. The reason for nontesting was not noted in the patient file and, therefore, could not be retrieved. The only exception was that in 29 patients (21.5\%) with 'known cognitive problems', or 'not able to complete the questionnaire in a reliable way' the MoCA and HADS were not assessed.

Table 1 The number of patients assessed for each specific test of the assessment battery

\begin{tabular}{ll}
\hline Specific test & $\begin{array}{l}\text { Number of } \\
\text { patients assessed } \\
(n, \%)\end{array}$ \\
\hline Hand grip dynamometry & $74(54.4 \%)$ \\
TCST & $86(64.0 \%)$ \\
BI & $70(51.5 \%)$ \\
MOCA & $38(27.9 \%)$ \\
MOCA blind & $8(5.9 \%)$ \\
Swallow screening & $53(39.0 \%)$ \\
HADS & $47(34.6 \%)$ \\
NRS2002 & $76(55.9 \%)$ \\
\hline
\end{tabular}

There were 29 patients (21.5\%) with 'known cognitive problems', or 'not able to complete the questionnaire in a reliable way' which were not assessed with the MOCA and HADS 
Table 2 shows the results of each specific test for the total population $(n=135)$ and the subgroup analysis of ICU vs. non-ICU patients and those aged $<70$ years vs. those aged $\geq 70$ years.

Assessment of the studied population revealed a high prevalence of impairments. Physical assessment showed that $72 / 87(82.8 \%)$ of the tested patients could not complete the FTSTST within $14 \mathrm{~s}$ while $32 / 74(43.2 \%)$ had a diminished HGF ( $<80 \%$ predicted value). Median BI was 10/20 [IQR 4.0-18.0] while swallow screening was impaired in only $3 / 53(5.7 \%)$ patients. MoCA scores were impaired in $21 / 38(55.2 \%)$ and HADS was aberrant in 22/47 (46.8\%). NRS 2002 evaluation showed that 24/76 (31.6\%) had a risk of malnutrition.

Patients aged $\geq 70$ years had more difficulty performing the FTSTST within $14 \mathrm{~s}$ compared to the group $<70$ years (4/45; $(8.9 \%)$ vs. 11/26; $(42.3 \%)$, respectively, $p=0.019)$.
The BI was lower in patients aged $\geq 70$ years, 8.0 [4.0-11.0], compared with those aged $<70$ years, 15.0 [7.5-19.0], $p=0.005$. There were more impaired MoCA scores in the group aged $\geq 70$ years $(11 / 13,84.6 \%, p=0.036)$ compared to those aged $<70$ years $(11 / 25,44.0 \%)$ with a difference in median MoCA scores, 21.0 [11.0-24.5] versus 26.0 [21.0-27.0], respectively, $p=0.029$.

ICU patients had a lower HGF (\%predicted value) compared to non-ICU patients, $69.8 \pm 23.1$ versus $93.7 \pm 30.7$, respectively $(p=0.001)$, with a mean difference $(95 \% \mathrm{CI})$ of $23.9 \pm 6.4$ and an OR $(95 \% \mathrm{CI})=4.25(1.51-11.98)$. The risk of malnutrition according to the NRS 2002 was more pronounced in ICU patients $(8 / 15,53.3 \%)$ compared to nonICU patients $(16 / 61 ; 26.2 \%)$ but this difference did not reach statistically significance $(p=0.063)$. There were no differences in MOCA scores between ICU, 26.0 [19.0-27.0], and non-ICU patients, 23.0 [17.0-27.0], $p=0.075$.

Table 2 Analysis of the results of the specific tests of the assessment battery

\begin{tabular}{|c|c|c|c|c|c|c|c|}
\hline & $\begin{array}{l}\text { Total population } \\
N=135\end{array}$ & $\begin{array}{l}\text { Age }<70 \text { years } \\
N=62\end{array}$ & $\begin{array}{l}\text { Age } \geq 70 \text { years } \\
N=73\end{array}$ & $p$ value & $\begin{array}{l}\mathrm{ICU} \\
N=33\end{array}$ & $\begin{array}{l}\text { Non-ICU } \\
N=102\end{array}$ & $P$ value \\
\hline \multicolumn{8}{|l|}{ Physical domain } \\
\hline $\begin{array}{l}\text { HGF (best side; \%predicted } \\
\text { value) }\end{array}$ & $85.9 \pm 30.5$ & $81.6 \pm 28.1$ & $89.6 \pm 32.3$ & .268 & $69.8 \pm 23.1$ & $93.7 \pm 30.7$ & $0.001 *$ \\
\hline $\begin{array}{l}\text { HGF }<80 \% \text { predicted value } \\
\quad(\mathrm{N}, \%)\end{array}$ & $32 / 74(43.2 \%)$ & $18 / 34(52.9 \%)$ & $14 / 40(35.0 \%)$ & .159 & $16 / 24(66.7 \%)$ & $16 / 50(32.0 \%)$ & $0.006^{*}$ \\
\hline FTSTST (seconds) $^{\mathrm{b}}$ & $14.5[10.3-18.8]$ & $13.0[9.0-16.0]$ & $17.0[11.5-20.0]$ & .185 & $16.0[11.0-27.5]$ & $14.0[10.0-18.0]$ & 0.173 \\
\hline $\begin{array}{l}\text { FTSTST } \geq 14 \text { or impossible to } \\
\text { perform }\end{array}$ & $71 / 86(82.6 \%)$ & $26 / 37(70.3 \%)$ & $45 / 49(91.8 \%)$ & $.019 *$ & $22 / 25(88.0 \%)$ & $49 / 61(80.3 \%)$ & 0.537 \\
\hline \multicolumn{8}{|l|}{ Functional domain } \\
\hline Barthel index ${ }^{b}$ & $10.0[4.0-18.0]$ & $15.0[7.5-19.0]$ & $8.0[4.0-11.0]$ & $.005^{*}$ & $9.0[4.0-16.0]$ & $10.0[4.0-18.0]$ & 0.763 \\
\hline Swallow screening impaired & $3 / 53(5.7 \%)$ & $1 / 27(3.7 \%)$ & $2 / 26(7.7 \%)$ & .610 & $2 / 19(10.5 \%)$ & $1 / 34(2.9 \%)$ & 0.290 \\
\hline \multicolumn{8}{|l|}{ Cognitive domain } \\
\hline MOCA $^{\mathrm{b}}$ & $23.0[18.8-27.0]$ & $26.0[21.0-27.0]$ & $21.0[11.0-24.5]$ & $.029 *$ & $26.0[19.0-27.0]$ & $23.0[17.0-27.0]$ & 0.663 \\
\hline MOCA $<26$ & $21 / 38(55.2 \%)$ & $11 / 25(44.0 \%)$ & $11 / 13(84.6 \%)$ & $.036^{*}$ & $7 / 15(46.7 \%)$ & $14 / 23(60.9 \%)$ & 0.509 \\
\hline MOCA Blind $^{\mathrm{a}}$ & $13.0 \pm 4.3$ & $11.5 \pm 2.1$ & $13.8 \pm 4.8$ & .545 & $15.0 \pm 2.8$ & $12.7 \pm 4.7$ & 0.545 \\
\hline MOCA Blind $<18$ & $7 / 8(87.5 \%)$ & $2 / 2(100.0 \%)$ & $5 / 6(83.3 \%)$ & 1 & $2 / 2(100.0 \%)$ & $5 / 6(83.3 \%)$ & 1 \\
\hline \multicolumn{8}{|l|}{ Psychological domain } \\
\hline HADS-D ${ }^{b}$ & $6.0[2.0-9.0]$ & $5.5[2-10.3]$ & $6[4.0-8.5]$ & .569 & $5.5[2.0-9.3]$ & $6.0[2.0-10.5]$ & 0.583 \\
\hline HADS-A ${ }^{b}$ & $6.0[3.0-9.0]$ & $6.0[3.0-9.0]$ & $6[3.5-9.0]$ & .855 & $6.0[3.0-9.0]$ & $6.0[3.0-10.0]$ & 0.598 \\
\hline $\mathrm{HADS}^{\mathrm{b}}$ & $11.0[6.0-17.0]$ & $10.5[4.8-17.5]$ & $11[8.5-18.0]$ & .622 & $10.5[5.8-15.5]$ & $11.0[6.0-21.0]$ & 0.561 \\
\hline HADS-D $>7$ & $16 / 47(34.0 \%)$ & $10 / 26(38.5 \%)$ & $6 / 21(28.6 \%)$ & .547 & $6 / 18(33.3 \%)$ & $10 / 29(34.5 \%)$ & 1 \\
\hline HADS-A > 7 & $16 / 47(34.0 \%)$ & $9 / 26(34.6 \%)$ & $7 / 21(38.9 \%)$ & 1 & $6 / 18(33.3 \%)$ & $10 / 29(34.5 \%)$ & 1 \\
\hline \multicolumn{8}{|l|}{ Nutritional domain } \\
\hline NRS 2002 risk of malnutrition & $24 / 76(31.6 \%)$ & $10 / 32(31.3 \%)$ & $14 / 44(31.8 \%)$ & 1 & $8 / 15(53.3 \%)$ & $16 / 61(26.2 \%)$ & 0.063 \\
\hline
\end{tabular}

Results are displayed for the entire tested population and for subgroup analysis of ICU vs. non-ICU and $<70$ years vs. $\geq 70$-years-old. Statistical significant $\mathrm{p}$ values are marked with an asterisk

${ }^{\text {a}}$ Normally distributed data

${ }^{\mathrm{b}}$ Non-normally distributed data 


\section{Discussion}

The results of the assessment show that physical, functional, cognitive, nutritional, and psychological impairments are highly prevalent in the group of COVID-19 patients, both in ICU and non-ICU patients.

We found a higher median age in the non-ICU versus our ICU population, likely to be explained by a possible selection bias. The Clinical Frailty Scale [24] was registered for patients with an age $\geq 70$ years. Patients with a higher frailty score, which are more likely to be older patients, were less likely to be admitted to the ICU. Also we did not assess patients $<70$ years who were not hospitalized for more than 7 days as this group seemed less relevant from a clinical perspective and resources had to be targeted towards groups with the highest risk of developing impairments. This age difference has to be taken into account when comparing the data of ICU vs. non-ICU groups.

Physical impairments are highly prevalent as shown by the FTSTST time and the hand grip dynamometry values. In addition to the physical impairments, the median BI indicates that the level of self-dependency is limited [25]. Both findings are in concordance with the study of Belli et al. [26] and Paneroni et al. [27].

The HGF was the only test that was significantly lower in ICU patients compared to non-ICU patients with a difference of $(95 \% \mathrm{CI})$ of $23.9 \pm 6.4 \%$ predicted value. This could be explained by the fact that the ICU patients had a high risk of developing ICU-acquired weakness [28, 29]. The percentage of aberrant swallowing screenings of COVID-19 patients on ICU was comparable to other nonCOVID-19 ICU studies [30].

Older adults were less able to perform the FTSTST within $14 \mathrm{~s}$. Physical impairment might be explained by disease itself, but quarantine or isolation measures might also lead to diminished physical activity and thus reducing physical strength [31]. The BI was lower in the older adults. On one hand this might be explained by the fact that physical and cognitive functions are also more impaired in this group, on the other hand it is possible that they had a lower premorbid BI because they contained more nursing home residents. These premorbid BI were not available in the patients' files.

Cognitive impairment was frequent in our cohort as reflected in the high proportion of patients with impaired MoCA scores. More studies are investigating the possible impact of COVID-19 on cognitive function. Zhou et al. [32] investigated 29 recovered COVID-19 patients and found that even after recovery, they performed less on online neuropsychological tests compared to healthy controls. The inflammatory response, hypercoagulability, neurotropic characteristics of the virus, or post-infectious immune mediated processes are all possible threats of COVID-19 to the central nervous system which could lead to several problems (e.g. stroke, hypoxia, delirium, etc.) that can diminish cognitive function [33-35]. There was no significant difference in the number of impaired MoCA scores between ICU and non-ICU patients. Literature concerning post-intensive care syndrome (PICS) shows that patients with prolonged ICU stay have high risk of impairments on neuropsychological test. Specifically for ARDS survivors, some studies show up to $71 \%$ of the patients having impairments on the neuropsychological tests [36], mainly problems with memory, executive function, language, attention, and visuospatial abilities [37]. The fact that the MoCA scores are comparable with non-ICU population might be explained by the fact that the non-ICU population was older. Our data already showed that older adults had a significantly higher number of impaired MoCA scores. This older non-ICU population is more likely to experience delirium [38] and/or have other causes of pre-existing cognitive impairments present which are more prevalent in the older adult population (e.g. delirium, vascular dementia, etc.) which might have been previously unknown/undiagnosed.

The HADS was aberrant in almost half of the tested patients. These results are in line with results of a systematic review concerning mental health consequences caused by COVID-19 [39] and reinforce the need for further psychological evaluation and follow-up if deemed necessary. There was no significant difference between ICU or nonICU patients concerning HADS scores. This is not completely unexpected as studies have shown that the levels of anxiety and depression were higher in the general population (non-infected people) compared to before the COVID-19 epidemic [39].

The risk of malnutrition according to the NRS 2002 was present in almost $1 / 3$ of the tested population. It was more pronounced in ICU patients compared to non-ICU patients but this difference did not reach statistical significance.

\section{Study limitations}

There are some limitations to this retrospective study. The number of patients for some specific tests was low and these numbers diminish even further with subgroup analyses. The low number of participants for some tests might impair the statistical power of the observations. However, this paper primarily contributes with an important descriptive component concerning the assessment of the functional status of COVID-19 patients. The reason why some patients were not tested could not be retrieved from the patients' files. It is possible that patients who performed better were tested less because this would have less impact on clinical decision making and resources might have been 
needed elsewhere. There was no information concerning the premorbid function of these patients. This could have impacted the results as patients with a lower premorbid function which was known might have been tested less because they were already care-dependent and the assessment would have changed less in the organization of their discharge care.

Despite its limitations, this paper offers an important basis for the assessment COVID-19 patients. Others have indicated the need for a broad evaluation of the COVID19 patient. For example, Klok et al. [40] already suggested a simple post-COVID tool assessment to measure the functional status over time using ordinal patient reported outcome measures. In contrast to proposed tool of Klok et al., we used a combination of patient reported outcome measures (e.g. HADS, etc.) and objective outcome measures (e.g. FTSTST, HGF, etc.) which might provide more detailed information. The British Society of Rehabilitation Medicine developed a framework for the assessment of post-ICU (COVID-19) patients [41]. However, based on our findings we would recommend to assess both ICU and non-ICU patients since both have high prevalence of physical, cognitive, psychological, and functional impairments. We, therefore, believe that this paper provides a base for future studies to optimise an assessment to evaluate the needs of (COVID-19) patients and install adequate follow-up trajectories. Future (prospective) studies should focus on an assessment of the patients functioning, ideally comparing this with premorbid functioning, which should lead to the optimal care/rehabilitation.

\section{Conclusion}

The high prevalence of physical, cognitive, psychological, nutritional, and functional impairments in hospitalized COVID-19 patients, both ICU as non-ICU patients, indicates that a pre-discharge assessment of impairments is imperative. Based on our data, we would recommend a minimal screening of physical, functional, cognitive, psychological and nutritional impairments. The high prevalence of impairments in multiple domains implies that adequate care and/ or multidisciplinary rehabilitation and follow-up after discharge seem to be essential for these patients.

Screening for possible impairments in different domains allows us to evaluate which type of rehabilitation is most adequate for each patient. This paper gives a short, workable assessment composed with known outcome measures to assess different domains of COVID-19 patients. The results of this assessment can be used to plan further care of the patient within the possibility of the hospital/home environment.
Funding No funding was present.

Availability of data and material Data are available on reasonable request to the corresponding author.

\section{Declarations}

Conflict of interest None of the authors has a conflict of interest.

Ethics approval Approval of the Ethical Committee Research UZ/KU Leuven was obtained for this retrospective analysis (S-number 64591). The need for an informed consent of participants was waived by the Ethical Committee.

Informed consent All authors consent for publication.

\section{References}

1. Fauci AS, Lane HC, Redfield RR (2020) Covid-19_navigating the uncharted. N Engl J Med 382(13):1268-1269

2. België FoBZ. BEVOLKINGSCIJFERS PER PROVINCIE EN PER GEMEENTE OP 1 JANUARI 2020 2020. https://www. ibz.rrn.fgov.be/fileadmin/user_upload/fr/pop/statistiques/popul ation-bevolking-20200101.pdf. Accessed 11 Oct

3. Sciensano. COVID-19-WEKELIJKS EPIDEMIOLOGISCH BULLETIN VAN 9 APRIL 2020 2020. https://covid-19.scien sano.be/sites/default/files/Covid19/COVID-19_Daily\%20rep ort_20200409\%20-\%20NL.pdf. Accessed 11 Oct

4. Negrini S, Ferriero G, Kiekens C, Boldrini P (2020) Facing in real time the challenges of the COVID-19 epidemic for rehabilitation. Eur J Phys Rehabil Med 56(3):313-315

5. Stam HJ, Stucki G, Bickenbach J, European Academy of Rehabilitation M (2020) Covid-19 and post intensive care syndrome: a call for action. J Rehabil Med. 52(4):jrm00044

6. Perrotta F, Corbi G, Mazzeo G, Boccia M, Aronne L, D'Agnano $\mathrm{V}$ et al (2020) COVID-19 and the elderly: insights into pathogenesis and clinical decision-making. Aging Clin Exp Res 32(8):1599-1608

7. Van Aerde N, Van den Berghe G, Wilmer A, Gosselink R, Hermans G, Consortium C- (2020) Intensive care unit acquired muscle weakness in COVID-19 patients. Intensive Care Med. 46(11):2083-2085

8. Frontera W, Gimigliano F, Melvin J, Li J, Li L, Lains J et al (2019) ClinFIT: ISPRM's universal functioning information tool based on the WHO's ICF. J Int Soc Phys Rehabil Med 2(1):19-21

9. Medicine ISPaR. Survey: proposal ClinFIT Covid-19 $2020 \mathrm{https} / / /$ www.isprm.org/survey-proposal-clinfit-covid-19/. Accessed 11 Oct

10. Spruit MA, Holland AE, Singh SJ, Tonia T, Wilson KC, Troosters T (2020) COVID-19: interim guidance on rehabilitation in the hospital and post-hospital phase from a European Respiratory Society and American Thoracic Society-Coordinated International Task Force. Eur Respir J 56:2002197

11. Carotti M, Salaffi F, Sarzi-Puttini P, Agostini A, Borgheresi A, Minorati D et al (2020) Chest CT features of coronavirus disease 2019 (COVID-19) pneumonia: key points for radiologists. Radiol Med 125(7):636-646 
12. Bohannon RW (2006) Reference values for the five-repetition sit-to-stand test: a descriptive meta-analysis of data from elders. Percept Mot Skills 103(1):215-222

13. Hermans G, Clerckx B, Vanhullebusch T, Segers J, Vanpee G, Robbeets $C$ et al (2012) Interobserver agreement of Medical Research Council sum-score and handgrip strength in the intensive care unit. Muscle Nerve 45(1):18-25

14. Mahoney FI, Barthel DW (1965) Functional evaluation: the barthel index. Md State Med J 14:61-65

15. Martino R, Silver F, Teasell R, Bayley M, Nicholson G, Streiner DL et al (2009) The Toronto Bedside Swallowing Screening Test (TOR-BSST): development and validation of a dysphagia screening tool for patients with stroke. Stroke 40(2):555-561

16. Perry L (2001) Screening swallowing function of patients with acute stroke. Part one: identification, implementation and initial evaluation of a screening tool for use by nurses. J Clin Nurs 10(4):463-473

17. Perry L (2001) Screening swallowing function of patients with acute stroke. Part two: detailed evaluation of the tool used by nurses. J Clin Nurs 10(4):474-481

18. Nasreddine ZS, Phillips NA, Bedirian V, Charbonneau S, Whitehead V, Collin I et al (2005) The Montreal Cognitive Assessment, MoCA: a brief screening tool for mild cognitive impairment. J Am Geriatr Soc 53(4):695-699

19. Zigmond AS, Snaith RP (1983) The hospital anxiety and depression scale. Acta Psychiatr Scand 67(6):361-370

20. Kondrup J, Rasmussen HH, Hamberg O, Stanga Z, Ad Hoc EWG (2003) Nutritional risk screening (NRS 2002): a new method based on an analysis of controlled clinical trials. Clin Nutr 22(3):321-336

21. Mathiowetz V, Kashman N, Volland G, Weber K, Dowe M, Rogers S (1985) Grip and pinch strength: normative data for adults. Arch Phys Med Rehabil 66(2):69-74

22. Pakhale S, Bshouty Z, Marras TK (2009) Comparison of per cent predicted and percentile values for pulmonary function test interpretation. Can Respir J 16(6):189-193

23. Guralnik JM, Ferrucci L, Pieper CF, Leveille SG, Markides KS, Ostir GV et al (2000) Lower extremity function and subsequent disability: consistency across studies, predictive models, and value of gait speed alone compared with the short physical performance battery. J Gerontol A Biol Sci Med Sci 55(4):M221-M231

24. Rockwood K, Song X, MacKnight C, Bergman H, Hogan DB, McDowell I et al (2005) A global clinical measure of fitness and frailty in elderly people. CMAJ 173(5):489-495

25. Wade DT, Hewer RL (1987) Functional abilities after stroke: measurement, natural history and prognosis. J Neurol Neurosurg Psychiatry 50(2): 177-182

26. Belli S, Balbi B, Prince I, Cattaneo D, Masocco F, Zaccaria S et al (2020) Low physical functioning and impaired performance of activities of daily life in COVID-19 patients who survived hospitalisation. Eur Respir J 56(4):2002096

27. Paneroni M, Simonelli C, Saleri M, Bertacchini L, Venturelli M, Troosters T et al (2020) Muscle strength and physical performance in patients without previous disabilities recovering from COVID19 pneumonia. Am J Phys Med Rehabil 100:105-109

28. Appleton RT, Kinsella J, Quasim T (2015) The incidence of intensive care unit-acquired weakness syndromes: a systematic review. J Intensive Care Soc 16(2):126-136
29. Latronico N, Tomelleri G, Filosto M (2012) Critical illness myopathy. Curr Opin Rheumatol 24(6):616-622

30. Schefold JC, Berger D, Zurcher P, Lensch M, Perren A, Jakob SM et al (2017) Dysphagia in mechanically ventilated ICU patients (DYnAMICS): a prospective observational trial. Crit Care Med 45(12):2061-2069

31. Kirwan R, McCullough D, Butler T, de Heredia FP, Davies IG, Stewart C (2020) Sarcopenia during COVID-19 lockdown restrictions: long-term health effects of short-term muscle loss. Geroscience 42(6): 1547-1578

32. Zhou H, Lu S, Chen J, Wei N, Wang D, Lyu H et al (2020) The landscape of cognitive function in recovered COVID-19 patients. J Psychiatr Res 129:98-102

33. Koralnik IJ, Tyler KL (2020) COVID-19: a global threat to the nervous system. Ann Neurol 88(1):1-11

34. Cothran TP, Kellman S, Singh S, Beck JS, Powell KJ, Bolton CJ et al (2020) A brewing storm: the neuropsychological sequelae of hyperinflammation due to COVID-19. Brain Behav Immun 88:957-958

35. Raman B, Cassar MP, Tunnicliffe EM, Filippini N, Griffanti L, Alfaro-Almagro F et al (2020) Medium-term effects of SARS$\mathrm{CoV}-2$ infection on multiple vital organs, exercise capacity, cognition, quality of life and mental health, post-hospital discharge. medRxiv. 395:497

36. Pfoh ER, Chan KS, Dinglas VD, Girard TD, Jackson JC, Morris PE et al (2015) Cognitive screening among acute respiratory failure survivors: a cross-sectional evaluation of the Mini-Mental State Examination. Crit Care 19:220

37. Inoue $S$, Hatakeyama J, Kondo $Y$, Hifumi $T$, Sakuramoto $H$, Kawasaki T et al (2019) Post-intensive care syndrome: its pathophysiology, prevention, and future directions. Acute Med Surg 6(3):233-246

38. Kennedy M, Helfand BKI, Gou RY, Gartaganis SL, Webb M, Moccia JM et al (2020) Delirium in older patients with COVID19 presenting to the Emergency Department. JAMA Netw Open 3(11):e2029540

39. Vindegaard N, Benros ME (2020) COVID-19 pandemic and mental health consequences: systematic review of the current evidence. Brain Behav Immun 89:531-542

40. Klok FA, Boon G, Barco S, Endres M, Geelhoed JJM, Knauss S et al (2020) The Post-COVID-19 Functional Status scale: a tool to measure functional status over time after COVID-19. Eur Respir J 56(1):2001494

41. Collaborative NP-ICR (2020) Responding to COVID-19 and beyond: a framework for assessing early rehabilitation needs following treatment in intensive care. In: Society BSoRMaIC, editor, p 36

Publisher's Note Springer Nature remains neutral with regard to jurisdictional claims in published maps and institutional affiliations. 